\title{
CASOS DE CONTAMINACIÓN POR INTRUSIÓN SALINA EN ACUÍFEROS COSTEROS DE LA PENÍNSULA DE NICOYA (COSTA RICA)
}

\author{
Federico Arellano ${ }^{1)} \&$ Asdrúbal Vargas ${ }^{2)}$ \\ 1) Instituto Costarricense de Acueductos y Alcantarillados \\ 2) Escuela Centroamericana de Geología
}

(Recibido 4/9/2000; Aceptado 13/12/2000)

\begin{abstract}
The coastal aquifers of the north Pacific region of Costa Ricas are being used to supply the water for tourism, agriculture, and to meet the needs of the local population. The hydrodynamic characteristics of these areas are not well known, although the production of water from these aquifers is critical given the increasing demand for the water. The zones under investigation are located in a moderately to very dry tropical region causing there to be a limited the supply of the water resource.

Twenty four water reservoirs were identified in the Nicoya Península of Costa Rica containing discharge wells with a variable production of 0,08 to 20 liters per second, a thickness of 10 to 30 meters, and a transmissivity of 2,5 to 2200 square meter per day. Compared to other volcanic continental aquifers in the region, these aquifers have a moderate potential of water production. The over-explotation and the reduction of the recharge caused the drawdown of the water level of many wells and provoked salt intrusion in 15 wells.

To adequately manage the groundwater resource, it is neccesary to rationalize the comsumption of the water based upon its use. Potable water usage must be limited to drinking and other necessary household activities. Whereas recycled water can be used to meet the needs of other activities.
\end{abstract}

RESUMEN: Los acuíferos costeros del Pacífico Norte de Costa Rica están siendo usados para abastecer de agua a las actividades turísticas, agrícolas, y para suplir las necesidades de la población local. Las características hidrodinámicas de estos acuíferos no son bien conocidas, aunque la producción se está incrementando debido al aumento de la demanda. Las zonas de investigación tienen un clima de seco a moderadamente seco provocando una recarga y una escorrentía limitada de agua.

Se identificaron 24 acuíferos con pozos de producción variable desde 0,08 a 20 litros por segundo, con un espesor desde 5 a 30 metros y una transmisividad de 2,5 a 2200 metros cuadrados por día. Comparados con otros acuíferos de tipo continental de la región, estos acuíferos tienen un potencial de producción de agua moderado. La sobre-explotación y la disminución de la recarga de estos acuíferos ha provocado la contaminación por intrusión salina de al menos 10 pozos con la consecuente pérdida de calidad. El manejo adecuado del recurso hídrico subterráneo en esta zona implica la utilización racional del recurso, utilizando agua de buena calidad solo donde se requiere, y aumentando las prácticas de conservación agroforestal para aumentar la retención de la humedad del suelo y aumentar la recarga al acuífero.

\section{INTRODUCCIÓN}

El objetivo de este trabajo es caracterizar los principales acuíferos costeros de la península de Nicoya, así como estudiar las interacciones de agua dulce y salada, y los indicios de contaminación por intrusión salina debido a la sobreexplotación. Estos acuíferos están siendo explotados para abastecer de agua a las actividades turísticas, ganaderas y para dotar de agua potable 


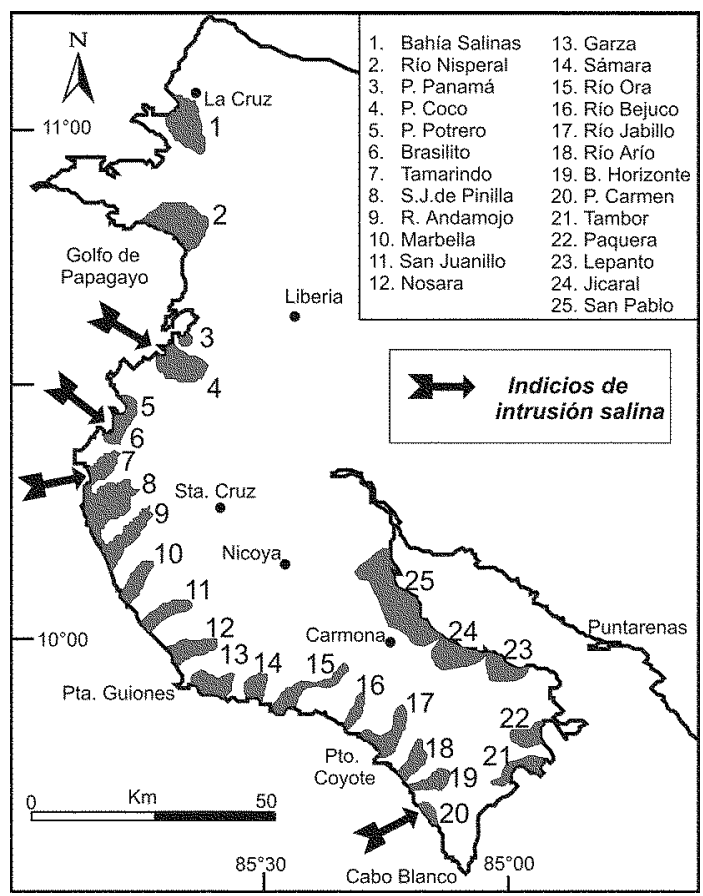

Fig. 1: Mapa de ubicación de los acuíferos costeros del Pacífico Norte de Costa Rica: 1. Bahía Salinas; 2. Río Nisperal; 3. P. Panamá. 4. P Coco; 5. P. Potrero; 6. Brasilito 7. Tamarindo; 8. San José de Pinilla; 9. Río Andamojo; 10. Marbella; 11. San Juanillo; 12. Nosara; 13. Garza; 14. Sámara; 15. Río Ora; 16. Río Bejuco; 17. Río Jabillo; 18. Río Arío; 19. B. Horizonte; 20. Tambor; 21. Paquera; 22. Lepanto; 23 Jicaral; 24 San Pablo.

a la población. Por otro lado la zona es una de las más secas de Costa Rica y el recurso hídrico es muy valioso. Se han identificado 24 zonas acuíferas, que se encuentran principalmente en la zona costera externa de la península de Nicoya (Fig. 1). Algunos estudios sobre los acuíferos costeros de la península de Nicoya han sido llevados a cabo por Chávez (1980), Herrera (1980), Protti (1981), Mora (1982) y Arredondo et al. (1992).

Como metodología de trabajo se han recopilado datos sobre conductividades eléctricas en más de 50 pozos excavados y perforados, obtenidos en una campaña de medición que se realizó durante 1994 y 1999. Se han obtenido datos sobre la estratigrafía a partir de perforaciones realizadas por empresas privadas a partir de los cuales se han construido per- files hidrogeológicos. Además se ha revisado literatura e informes técnicos del SENARA y AyA, lo que ha permitido identificar los acuíferos costeros.

Varios autores mencionan evidencias de contaminación en acuíferos costeros del pacífico. Esto representa una llamada de alerta para tomar acciones a favor de la protección del recurso hídrico subterráneo. La intrusión salina resulta una condición bastante frecuente en acuíferos mal gestionados, lo cual implica un deterioro en la calidad del recurso en una región donde es de suma importancia para múltiples actividades (Astorga et al., 2000). El Estado de la Nación señala a la provincia de Guanacaste como una de las zonas de menor riqueza hídrica del país. En ella la segunda fuente de abastecimiento de agua, después de la superficial, corresponde a el agua subterránea contenida en los acuíferos. Gran parte de la península de Nicoya tiene escaza agua subterránea, lo mismo que la zona costera, en la cual es frecuente la presencia de agua salinizada. Algunos de los acuíferos que están amenazados por la intrusión salina y la sobre-explotación son playa Panamá, Hermosa, del Coco, Potrero, Brasilito y Jicaral. Losilla (1993) menciona contaminación por intrusión salina en Bello Horizonte, playa Cocal, playa Cabuya, playa Santa Teresa, playa Carmen, playa Cabuya, playa Pochote, playa Tambor y playa Tamarindo.

Se denomina intrusión de agua salada al movimiento permanente o temporal del agua salada tierra adentro, desplazando el agua dulce. El agua captada en un acuífero costero se contamina (se saliniza) cuando la porción activa de la captación se ve afectada por la zona de mezcla de agua dulce y agua salada o por la propia agua salada. Si dos líquidos son miscibles, tales como agua dulce y agua salada, no existe una interfase brusca sino que se pasa de un fluido a otro a través de una zona de mezcla. Esta zona de mezcla, es también llamada zona de difusión o de transición, refleja con intensidad variable las propiedades químicas e hidráulicas de cada uno de los líquidos originales y su anchura depende de la difusividad y dispersividad del medio y de las características del movimiento. El peso específico del agua dulce se puede tomar como 
$\mathrm{gd}=1000 \mathrm{~kg} / \mathrm{m}^{3} \mathrm{y}$ el peso específico del agua de mar se puede tomar como gs $=1025 \mathrm{~kg} / \mathrm{m}^{3}$; la viscosidad del agua marina es del orden de $30 \%$ mayor que la del agua dulce a igual temperatura. Los primeros estudios de la relación del agua dulce y del agua salada en regiones costeras se realizaron en Holanda y Alemania por Badon Ghyben (1889) y Herzberg (1901). Se basa en el equilibrio estático de columnas de agua de diferente densidad. Las hipótesis básicas son:

$1 \quad$ El flujo de agua dulce es casi horizontal y por lo tanto el potencial es constante a lo largo de cualquier vertical.

$1 \quad$ No existe flujo de agua salada.

$1 \quad$ La interfase es un plano, por tanto no existe zona de mezcla. En estas condiciones, en un punto cualquiera de la interfase debe equilibrarse la presión del agua dulce y del agua salada.

$\left(\mathrm{h}_{\mathrm{d}}+\mathrm{z}\right) \gamma_{\mathrm{d}}=\mathrm{z} \gamma_{\mathrm{s}}$

siendo:

$h_{d}$ : cota sobre el nivel del mar del agua dulce en la vertical del punto A

Z: profundidad bajo el nivel del mar del punto A

$\gamma_{\mathrm{d}}$ : peso específico del agua dulce

$\gamma_{\mathrm{s}}$ : peso específico del agua salada

Así pues,

$\mathrm{z}=\frac{\gamma_{\mathrm{d}}}{\gamma_{\mathrm{s}}-\gamma_{\mathrm{d}}} * \mathrm{~h}_{\mathrm{d}}=\mathrm{h}_{\mathrm{d}} / \beta$

siendo

$\frac{1}{\beta}=\frac{\gamma_{d}}{\gamma_{s}-\gamma d}$

1/ $\beta$ varía de acuerdo con lo dicho entre 50 y 33 siendo el valor más frecuente 40. Ello quiere decir que la interfase se sitúa a una profundidad bajo el nivel del mar igual a 40 veces la cota del agua dulce sobre el nivel medio del mar en aquel punto. La máxima penetración de la cuña de agua salada viene limitada por el fondo impermeable del acuífe- ro, que se produce cuando hd $=$ zo $b$ siendo zo la profundidad de la base del acuífero.

\section{ACUÍFEROS COSTEROS}

Este es un grupo muy numeroso de acuíferos y son de gran importancia para abastecer de agua múltiples actividades que se desarrollan en las zonas costeras del Pacífico Norte (Cuadro 1). El agua subterránea contenida en estos acuíferos se extrae principalmente por medio de pozos verticales que tienen un diámetro promedio aproximado de $250 \mathrm{~mm}$. Además se emplean pozos excavados cuyo diámetro puede alcanzar hasta $1 \mathrm{~m}$., o se captan manantiales. Estos acuíferos reciben agua a través de las zonas de recarga en las partes altas de las montañas pero también admiten agua de los ríos. Por su parte la descarga natural de agua subterránea se da a través de los manantiales, o por la descarga hacia el mar. Los acuíferos más explotados son Nosara, Garza y Sámara, entre otros.

Estos acuíferos están formados por depósitos aluvionales que contienen gravas y arenas muy permeables (Cuadro 1), con espesores de 10-50 m, con caudales que varían de 1-50 1/s y cubiertos o intercalados con capas de arcilla de baja conductividad hidráulica (Fig 2). Chávez \& Herrera (1980) estiman espesores para el área de Brasilito entre 3 y $11 \mathrm{~m}$ mientras que de 15 a 60 $\mathrm{m}$ en la zona de playa Potrero. En ambas cuencas se realizaron pruebas de bombeo que indican valores de transmisividad de alrededor de $200 \mathrm{~m}^{2} / \mathrm{d}$. Por su parte Protti (1980) estima que la zona de más potencial hidrogeológico en la cuenca del río Andamojo se localiza entre las localidades de Veintisiete de Abril y Paraíso donde se pueden encontrar espesores de alrededor de $25 \mathrm{~m}$.

\section{AMENAZA DE CONTAMINACIÓN DE LOS ACUÍFEROS}

Existe evidencia de que ciertos acuíferos costeros han sido sobreexplotados en tal medida que los mismos se han salinizado a tal grado que su explotación se ha abandonado y se ha tenido que recurrir a la búsqueda de nuevas fuentes de 
Cuadro 1

Acuíferos costeros de la península de Nicoya

\begin{tabular}{|c|c|c|c|c|c|c|c|}
\hline No & Acuífero & $\mathrm{Q}(1 / \mathrm{s})$ & $\mathrm{E}(\mathrm{m})$ & Localidad & $\mathrm{T}(\mathrm{m} 2 / \mathrm{d})$ & $\mathrm{k}(\mathrm{m} / \mathrm{d})$ & S \\
\hline 1 & Bahía Salinas & $0,3-12$ & 15 & P. Soley & 37 & & \\
\hline 2 & R. Nisperal & $1-5$ & 10 & & & & \\
\hline 3 & Playa Panamá & $1-5$ & 20 & Playa Panamá & 246 & & \\
\hline 4 & El Coco & 0,5 & 10 & El Coco & 110 & & \\
\hline 5 & Potrero & $1-5$ & $15-60$ & Potrero & 200 & & \\
\hline 6 & Brasilito & 0,5 & $3-11$ & Brasilito & $3-200$ & 0,2 & \\
\hline 7 & Tamarindo & $2-6$ & 10 & Tamarindo & - & - & \\
\hline 8 & S. J. Pinilla & $1-5$ & 10 & S. J. Pinilla & 15 & 1,5 & - \\
\hline 9 & R. Andamojo & $1-5$ & 20 & R. Seco & 1080 & $6,0-43,0$ & 0,05 \\
\hline 10 & Marbella & $1-5$ & 10 & Marbella & - & - & - \\
\hline 11 & Sn. Juanillo & $2-5$ & 20 & Sn. Juanillo & 15 & 0,5 & 0,5 \\
\hline 12 & Nosara & 9 & 15 & Nosara & $28-169$ & 1,8 & 0,0004 \\
\hline 13 & Garza & $1-5$ & 10 & Garza & 295 & - & - \\
\hline 14 & Sámara & 1 & 10 & Sámara & $9-2115$ & 0,7 & 0,03 \\
\hline 15 & R. Ora & $1-5$ & 15 & Arbolito & - & - & - \\
\hline 16 & Bejuco & $1-5$ & 25 & Bejuco & 2246 & 89,9 & 0,13 \\
\hline 17 & R. Jabillo & $1-5$ & 10 & P. Coyote & & & \\
\hline 18 & Arío y Bongo & $1-5$ & 15 & San Lucas & & & \\
\hline 19 & B. Horizonte & $0,08-1,5$ & $<15$ & B.Horizonte & $3-5$ & 0,3 & 0,01 \\
\hline 20 & Tambor & $1-5$ & 20 & Cóbano & & & \\
\hline 21 & Paquera & $1-5$ & 20 & Paquera & 384 & & \\
\hline 22 & Lepanto & $1-10$ & 30 & Lepanto & & & \\
\hline 23 & Jicaral & $1-11$ & $<40$ & Jicaral & & & \\
\hline 24 & San Pablo & & & San Pablo & & & \\
\hline
\end{tabular}

Q: caudal promedio; E: espesor promedio; T: transmisividad, k: conductividad hidráulica; S: coeficiente de almacenamiento.

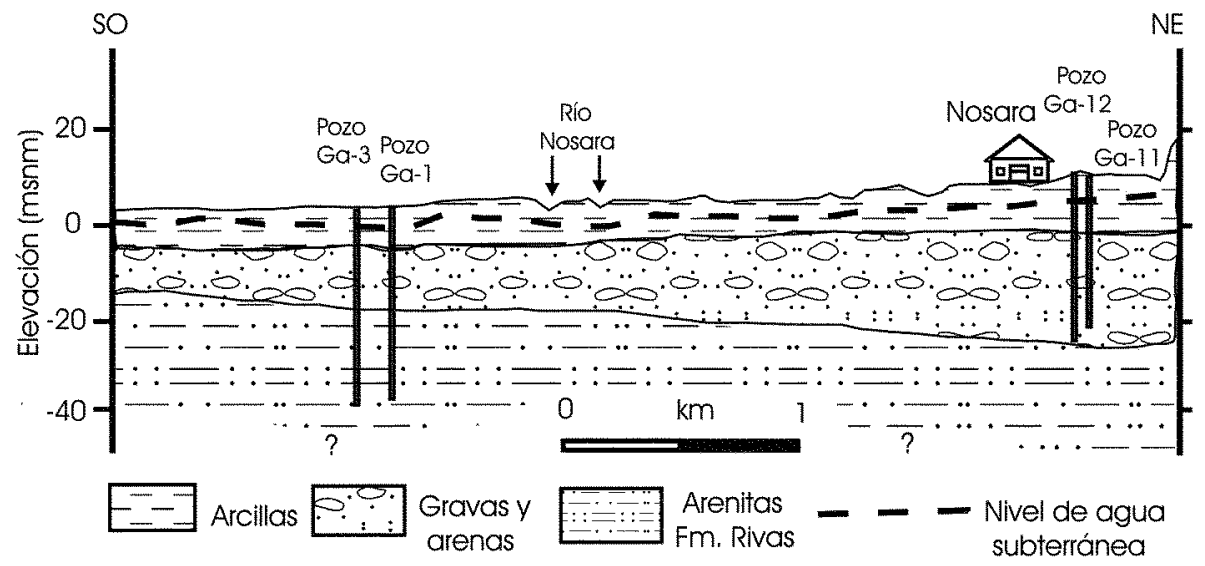

Fig. 2: Perfil hidrogeológico del acuífero de Nosara.

agua potable; tal es el caso de Flamingo. Se dice que los pozos que abastecían la zona turística se salaron, por lo que se hizo necesario perforar nuevos pozos a unos $4 \mathrm{~km}$ de playa Potrero, con lo cual solucionaron el problema. No obstante, por la ignorancia y el mal manejo del recurso hídrico provocaron daño al acuífero y será la propia naturaleza la encargada de revertirlo en un 


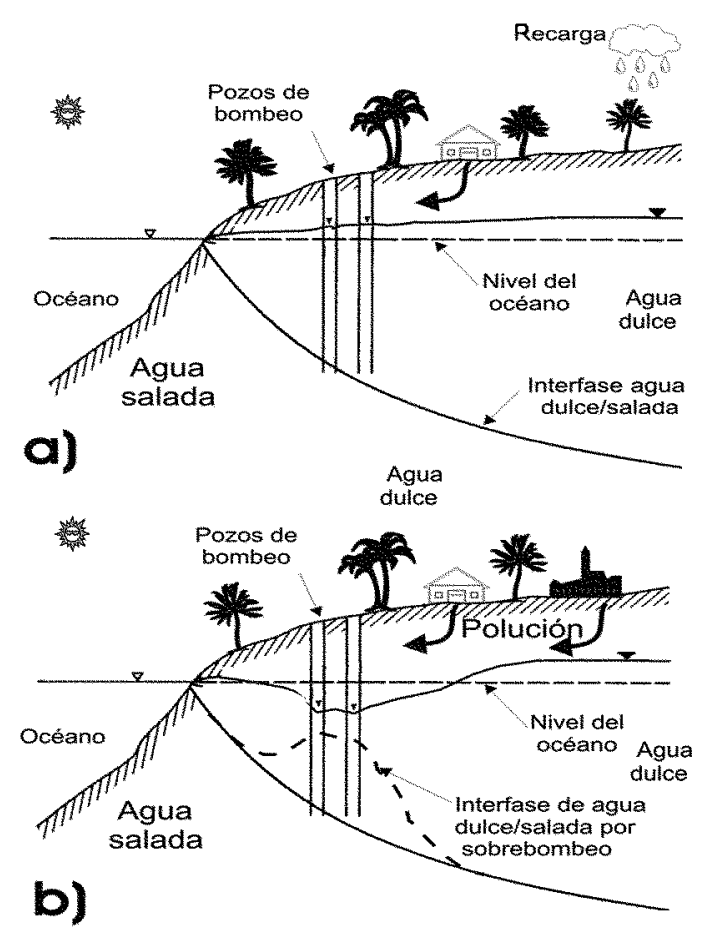

- Nivel del agua subterránea

Fig. 3: a) Esquema de interfase agua dulce- agua salada en situación natural; b) esquema de interfase agua dulce-agua salada en situación de sobrebombeo. plazo relativamente largo. Como este ejemplo, existen varios de la misma naturaleza, con la consecuente pérdida de la calidad del recurso hídrico. En la figura 3a se muestra el esquema de la interfase agua dulce-salada en la condición de bombeo racional, mientras que en la figura $3 \mathrm{~b}$ se muestra el cambio de posición de la interfase luego de un bombeo excesivo.

\section{CASO DE PLAYA GRANDE}

Playa Grande está ubicada entre las coordenadas 255-257 y 334-336 de la hoja topográfica Villareal, que forma parte de la bahía de Tamarindo y está constituida por una faja de arena y limo. Se encuentra limitado al este y norte por el estero Tamarindo y al sur y al oeste por el océano Pacífico. Existen ocho pozos de explotación con profundidades que varían entre 4,2 y 13,3 m y con niveles freáticos de 2,5 a $3 \mathrm{~m}$. De los ocho pozos, cuatro muestran contaminación por intrusión salina. Al observar la disposición de los pozos se nota que la zona de explotación recibe una recarga directa por precipitación y en menor grado por aportes desde el valle del río Matapalo que termina en el estero Tamarindo (Fig. 4).

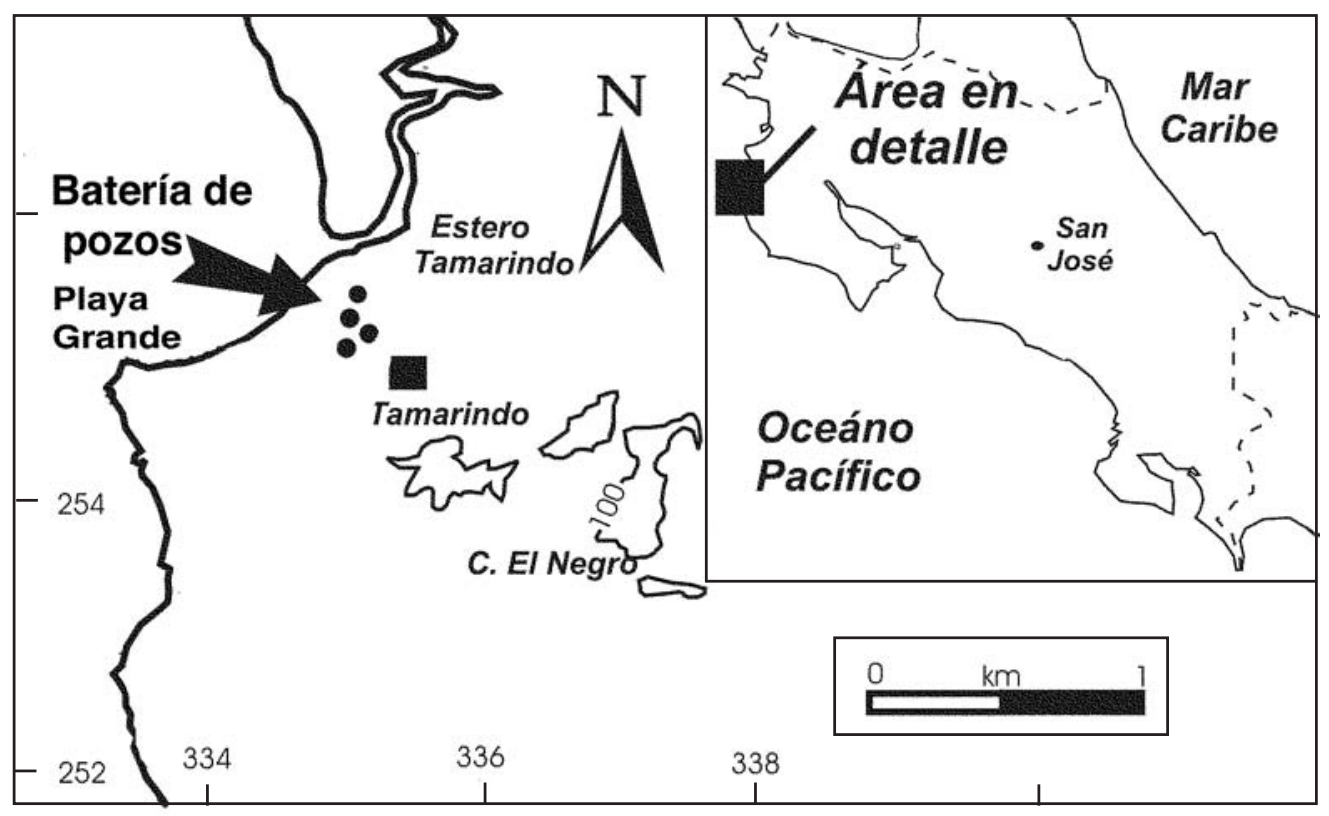

Fig. 4: Esquema de ubicación de Playa Grande y de la batería de pozos. algunos de ellos contaminados por intrusión salina. 


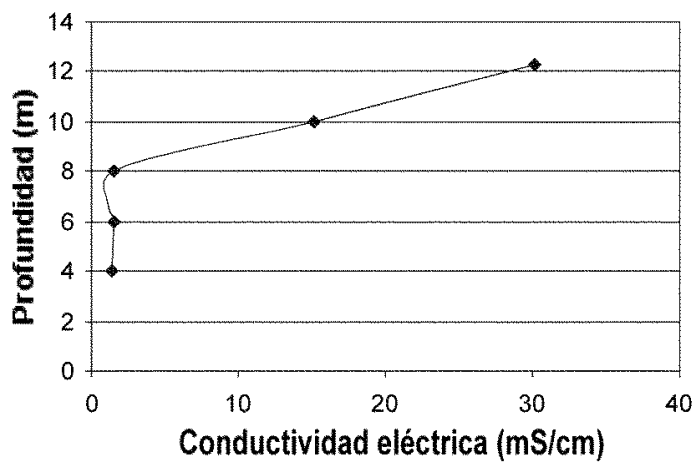

Fig. 5: Variación de la interfase agua dulce-salada mostrada por la variación de la conductividad eléctrica en el pozo P10 en Playa Grande.

Se midieron las conductividades eléctricas en dos pozos denominados P10 y P3 (Figs. 5 y 6), los cuales mostraron un gradiente de conductividad muy acusado con la profundidad en un caso a los 8 $\mathrm{m}$ y el otro a los $2 \mathrm{~m}$ evidenciando la interfase agua dulce-salada. Las posibles causas de la intrusión salina en esta zona se deben a la escasa recarga, la cual proviene en parte por la infiltración de lluvia. Además los pozos están muy cerca entre sí, favoreciendo la unión de los conos de abatimiento y el bombeo excesivo a provocado que el nivel de agua

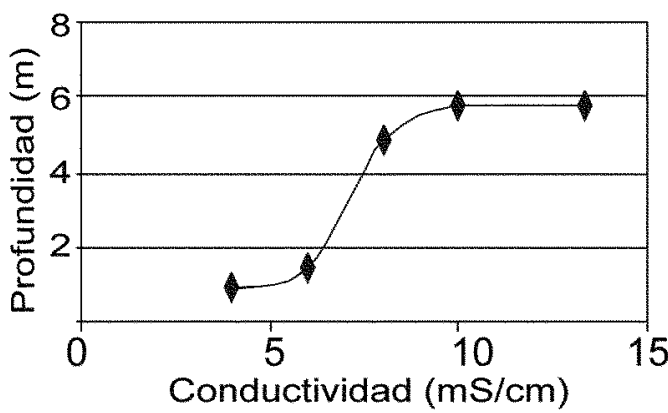

Fig. 6: Variación de la interfase agua dulce-salada mostrada por la variación de la conductividad eléctrica en el pozo P3 en Playa Grande.

en los pozos descienda más allá que el nivel de agua del mar favoreciendo la entrada de agua salada.

Además se tienen indicios de intrusión salina en playas del Coco, Brasilito y Playa Carmen. Los datos sobre conductividad eléctrica recogidos durante el año 1994 (Cuadro 2), indican valores que sobrepasan los $400 \mu \mathrm{S} / \mathrm{cm}$ que es considerado un valor límite. Durante esta campaña se midieron además valores de $\mathrm{pH}$, oxígeno disuelto y salinidad, sin embargo no se notaron valores fuera de las normas.

\section{Cuadro 2}

Características fisico-químicas de pozos en acuíferos costeros, medidos entre abril y mayo de 1994

\begin{tabular}{|c|c|c|c|c|c|c|c|c|c|c|}
\hline Hoja & Descripción & Lat. & Long. & NE. $(\mathrm{m})$ & P.(m) & $\mathrm{T} .(\mathrm{C})$ & C. $(\mathrm{mS} / \mathrm{cm})$ & $\mathrm{pH}$ & $\mathrm{O}_{2}(\mathrm{mg} / \mathrm{l})$ & S \% \\
\hline Matapalo & P. Brasilito & 267,3 & 340,5 & 3,9 & 6 & 31,4 & 0,318 & & & \\
\hline Matapalo & P. Brasilito & 267,6 & 340,8 & & 8 & 33 & 0,327 & & & \\
\hline Matapalo & P. Brasilito & 268,1 & 341,0 & & & 31,2 & 0,526 & & & \\
\hline Matapalo & P. Potrero & 270,0 & 343,1 & & 23 & 28,4 & 0,368 & & & \\
\hline Matapalo & P. Prieta & 272,4 & 341,7 & & 83 & 30,6 & 0,183 & & & \\
\hline Matapalo & P. Zapotal & 275,7 & 340,5 & 3,0 & 10 & 28,3 & 0,473 & & & \\
\hline C. Norte & P.Hermosa & 283,8 & 352,9 & 11,5 & 18 & 30,8 & 0,465 & & & \\
\hline C. Norte & V. Huetares & 284,3 & 352,8 & 8,0 & 40 & 30,3 & 0,449 & & & \\
\hline C, Norte & H. Velero & 284,5 & 352,8 & 4,3 & 30 & 30,5 & 0,692 & & & \\
\hline C. Norte & P.Hermosa & 284,8 & 352,9 & 3,2 & & 30,6 & 0,490 & & & \\
\hline C. Norte & P.Italiano & 286,5 & 356,8 & 6,2 & 30 & 30,8 & 0,410 & & & \\
\hline C. Norte & P.Panamá & 285,7 & 354,8 & 5,5 & & 29,3 & 0,427 & & & \\
\hline Tambor & Madrigales & 199,0 & 434,8 & Surg. & 28 & 28,8 & 0,490 & 7,12 & 1,46 & 0,02 \\
\hline Tambor & Reserva Curú & 197,9 & 433,4 & 2,7 & 8 & 32,6 & 0,487 & 7,00 & 3,54 & 0,02 \\
\hline Río Ario & P.Pochote & 193,3 & 426,8 & & & 28,5 & 0,470 & 7,45 & 6,2 & 0,01 \\
\hline
\end{tabular}


Continuación...

\begin{tabular}{|c|c|c|c|c|c|c|c|c|c|c|}
\hline Hoja & Descripción & Lat. & Long. & NE. ( m) & $\mathrm{P} .(\mathrm{m})$ & $\mathrm{T} .(\mathrm{C})$ & C. $(\mathrm{mS} / \mathrm{cm})$ & $\mathrm{pH}$ & $\mathrm{O}_{2}(\mathrm{mg} / \mathrm{l})$ & S \% \\
\hline Tambor & Tambor & 189,9 & 423,4 & 11,0 & & 28,2 & 0,477 & 7,33 & 2,01 & 0,01 \\
\hline Cabuya & P. Carmen & 179,4 & 410,2 & & 20 & 28,5 & 1,620 & 8,25 & 1,95 & 0,07 \\
\hline Cabuya & C. Chamorro & 180,9 & 408,4 & 5,0 & 8 & 27,7 & 0,582 & 7,07 & 4,8 & 0,02 \\
\hline Cabuya & P. Sta. Teresa & 181,2 & 408,1 & 7,7 & 26 & 34 & 0,778 & 7,07 & 2,19 & 0,03 \\
\hline Río Ario & P.Manzanillo & 187,4 & 403,5 & 3,7 & 6 & 28,7 & 0,737 & 7,16 & 5,3 & 0,03 \\
\hline Río Ario & B.Horizonte & 186,5 & 404,6 & 5,0 & 17 & 31,8 & 0,801 & 7,63 & 5,33 & 0,03 \\
\hline Río Ario & Bajos de Ario & 191,0 & 404,5 & 4,5 & 7 & 29,5 & 0,317 & 6,55 & 4,06 & 0,01 \\
\hline Río Ario & Bajos de Arío & 191,1 & 404,5 & 4,7 & & 29,7 & 0,178 & 6,57 & 2,04 & 0,01 \\
\hline C.Azul & Punta Islita & 204,4 & 382,7 & 5,2 & 10 & 30,7 & 0,257 & 6,78 & 1,35 & 0,01 \\
\hline C.Azul & Gan. Viales & 204,8 & 387,8 & 7,5 & 21 & 28,3 & 0,137 & 6,93 & 2,77 & 0 \\
\hline P. Coyote & P.de Bejuco & 204,5 & 388,5 & & 40 & 29,3 & 0,17 & 7,24 & 4,95 & 0 \\
\hline C.Azul & Pueblo Nuevo & 202,5 & 390,5 & 2,5 & & 28,8 & 0,17 & 7,28 & 3,92 & 0 \\
\hline C. Azul & Gno Garcia & 202,6 & 390,6 & 5,4 & 8 & 27,7 & 0,419 & 7,49 & 4,05 & 0,01 \\
\hline C. Azul & P. Carrillo & 205,8 & 373,8 & 3,7 & 30 & 26,3 & 0,305 & 7,27 & 4,77 & 0,01 \\
\hline Garza & Sámara & 207,0 & 369,1 & & 35 & & 0,224 & 7,20 & & \\
\hline Garza & Fin. Verde Bren & 207,6 & 370,1 & & & 27,8 & 0,270 & 7,28 & 1,88 & 0,01 \\
\hline Garza & Torito & 207,4 & 370,7 & 10,2 & & 29,3 & 0,464 & 7,38 & 4,67 & 0,01 \\
\hline Garza & Cangrejal & 209,1 & 366,7 & 7,0 & 19 & 29,2 & 0,578 & 7,30 & 2,3 & 0,02 \\
\hline Garza & Garza & 210,8 & 356,1 & 2,5 & 15 & 29,7 & 0,558 & 7,41 & 4,35 & 0,02 \\
\hline Garza & P.Guiones & 212,1 & 354,5 & 2,9 & & 28,5 & 0,595 & 7,35 & 2 & 0,02 \\
\hline Garza & P.Guiones & 211,9 & 354,4 & 3,2 & 7 & 28,2 & 0,554 & 7,25 & 3,17 & 0,02 \\
\hline Matapalo & P. De C. Rica & 267,1 & 339,2 & 3,6 & 4 & 29,2 & 0,404 & 7,14 & 3,23 & 0,04 \\
\hline Matapalo & Don Conrado & 267,6 & 341,0 & 7,9 & 8 & 30,4 & 0,311 & 7,01 & 3,94 & 0,01 \\
\hline Matapalo & P.Flamingo & 268,1 & 340,9 & 8,3 & & 31,1 & 0,373 & 7,03 & 5,12 & 0,01 \\
\hline Matapalo & T. Ramos & 267,5 & 340,4 & 3,7 & 4 & 29,4 & 0,287 & 7,16 & 3,61 & 0,01 \\
\hline Matapalo & Nina & 267,1 & 341,3 & & & 29,4 & 0,180 & 9,70 & 0,25 & \\
\hline Matapalo & Julio López & 267,2 & 341 & 8,6 & & 29,1 & 0,305 & 6,96 & 4,45 & 0,01 \\
\hline Matapalo & Mr Kelly & 270,1 & 343,1 & 3,1 & & 28,0 & 0,351 & 7,09 & 5,15 & 0,01 \\
\hline Matapalo & Mr Kelly & 269,9 & 343,1 & 3,1 & & 28,1 & 0,367 & 7,06 & 4,30 & 0,01 \\
\hline Matapalo & & 271,4 & 342,4 & 4,5 & & 28,0 & 0,466 & 7,33 & 5,45 & 0,01 \\
\hline Matapalo & Pozo AyA & 271,6 & 342,5 & 3,0 & & 29,2 & 0,426 & 7,17 & 4,25 & 0,01 \\
\hline Matapalo & & 271,5 & 343,2 & 11,3 & & 29,0 & 0,422 & 7,09 & 2,29 & 0,01 \\
\hline Matapalo & & 271,1 & 343,3 & 6,2 & & 29,5 & 0,377 & 7,03 & 2,85 & 0,01 \\
\hline Matapalo & & 272,3 & 341,6 & & & 29,4 & 0,145 & 9,37 & 2,10 & 0 \\
\hline
\end{tabular}

NE: Nivel estático, P: profundidad del pozo, T: temperatura, C: Conductividad, S: salinidad, Surg.: surgente.

\section{CONCLUSIONES}

En los acuíferos costeros los niveles del agua dulce a lo largo de la playa podrían descender en gran medida por una extracción masiva e incontrolada de agua. La extracción artificial intensiva de agua de estos acuíferos debe evitarse dentro de una faja de unos $500 \mathrm{~m}$ paralela a la playa.

Los acuíferos costeros presentan un potencial de rendimiento de agua moderado a bajo, mientras que la demanda aumenta, por lo que se deben establecer políticas de protección al recurso por parte de las municipalidades y de las entidades encargadas del recurso hídrico. Se han identificado varios acuíferos que muestran indicios de intrusión salina; sin embargo, es necesario continuar con los muestreos de agua para evaluar el alcance de la contaminación.

Se han identificado indicios de intrusión salina en playas del Coco, Brasilito y playa Carmen, los cuales, sin embargo, deben corroborarse por medio de mediciones sistemáticas de 
conductividad eléctrica. En el caso de playa Grande la recarga del acuífero se debe aumentar y disminuir la explotación, hasta que se recuperen los niveles y disminuya la salinidad por la disolución del agua de recarga.

\section{REFERENCIAS}

ARREDONDO, S., MORERA, S., PÉREZ, W. \& BOLAÑOS, A., 1992: Mapa hidrogeológico de la península de Nicoya. - Escala 1: 250 000, SENARA, San José.

ASTORGA, A., ASTORGA, Y., HERNÁNDEZ, G., MORA, J., TORRES, I., ALFONSO, F. \& LÓPEZ, R., 2000: Proyecto Sistemas Integrados de Gestión y Calidad Ambiental, SIGA. - 336 págs. Fundación para el desarrollo Urbano, San José.

CHÁVEZ, J., 1980: Aspectos geológicos de la cuenca del río Nimboyores, Guanacaste, Costa Rica. - 33 págs. Univ. de Costa Rica, San José [Tesis Lic.].

CHÁVEZ, J \& HERRERA, J., 1980: Proyecto de investigación de aguas subterráneas en la península de Nicoya. - 37 págs. Servicio Nacional de Aguas Subterráneas, Hidrogeología de la zona Brasilito-Potrero, San José.
HERRERA, J.A., 1980: Hidrogeología de la cuenca del río Andamojo, Santa Cruz, Guanacaste, Costa Rica. - 81 págs. Univ. de Costa Rica, San José [Tesis Lic.].

LOSILLA, M., 1993: Evidencias de amenaza a las aguas subterráneas de Costa Rica. - 23 págs. Taller de aguas subterráneas, Colegio Federado de Ingenieros, San José.

MORA, D., 1982: Contaminación de las aguas subterráneas en acuíferos de Costa Rica. - En: MATA, A., CHACÓN, B. \& RODRÍGUEZ, A. (eds.): Contaminación ambiental: un enfoque multidisciplinario. Ed. Tecnológica, Cartago:59-79.

O.N.U., 1973: Investigaciones de aguas subterráneas en zonas seleccionadas. - 64 págs. Nueva York.

PROYECTO ESTADO DE LA NACIÓN EN DESARROLLO HUMANO SOSTENIBLE, 1999: Estado de la Nación en Desarrollo Humano Sostenible. - Sexto Informe [ $1^{\mathrm{a}}$ ed.] 414 págs. San José.

PROTTI, R., 1980: Prospección geofísica por sísmica de refracción en la cuenca del río Andamojo, Santa Cruz, Guanacaste con fines hidrogeológicos.- 60 págs, SENARA [Informe interno \# 133].

PROTTI, R., 1981: Geología y geofísica con fines hidrogeológicos en la planicie costera Jicaral-Santa Rita, península de Nicoya. - 47 págs +5 mapas, Univ. de Costa Rica, San José [Tesis Lic]. 